\title{
Article \\ Effects of Surgical Treatment for Allergic Rhinitis on Sleep and Mental Health in Adolescents
}

\author{
Takayuki Nakagawa ${ }^{1,2,3, *}$, Eriko Ogino-Nishimura ${ }^{2,3}$ and Shinya Hiroshiba ${ }^{2}$ \\ 1 Department of Otolaryngology, Head and Neck Surgery, Graduate School of Medicine, Kyoto University, \\ Kyoto 6068507, Japan \\ 2 Kyoto ENT Surgicenter, Kyotanabe 6100355, Japan; e_ogino@ent.kuhp.kyoto-u.ac.jp (E.O.-N.); \\ hiro@hiroshiba.com (S.H.) \\ 3 Kyoto Nose and Allergy Clinic, Kyoto 6008216, Japan \\ * Correspondence: tnakagawa@ent.kuhp.kyoto-u.ac.jp; Tel.: +81-75-751-3346
}

check for updates

Citation: Nakagawa, T.; Ogino-Nishimura, E.; Hiroshiba, S. Effects of Surgical Treatment for Allergic Rhinitis on Sleep and Mental Health in Adolescents. Surgeries 2022, 3, 20-27. https://doi.org/10.3390/ surgeries3010005

Academic Editor: Cornelis F.M. Sier

Received: 13 November 2021

Accepted: 26 January 2022

Published: 28 January 2022

Publisher's Note: MDPI stays neutral with regard to jurisdictional claims in published maps and institutional affiliations.

Copyright: (C) 2022 by the authors. Licensee MDPI, Basel, Switzerland. This article is an open access article distributed under the terms and conditions of the Creative Commons Attribution (CC BY) license (https:// creativecommons.org/licenses/by/ $4.0 /)$.

\begin{abstract}
Allergic rhinitis (AR) is a common chronic condition that is usually treated medically. Adolescents form a unique population in which AR-associated symptoms are greater than those in adults or children, and are closely related to sleep and mental disorders. In the current study, a retrospective analysis was performed to illustrate the surgical effects of AR symptoms on sleep and mental disorders in adolescents. In 81 adolescents with AR symptoms refractory to medical management, the severity of the AR symptoms was correlated with that of sleep or mental disorders. As a standard surgical procedure, submucosal bony resection of inferior turbinates with posterior nasal neurectomy was performed and half of the subjects underwent septoplasty due to severe deviation of the nasal septum. The degree of improvement in AR-associated symptoms by surgical treatment was correlated with that of mental disorders in adolescent patients who had sleep and mental disorders preoperatively. Considering the impact of AR symptoms on the quality of life of adolescents, surgical treatment can be a potent option for the treatment of AR refractory to medical management in this population.
\end{abstract}

Keywords: allergic rhinitis; endoscopic surgery; mental health; nasal obstruction; sleep disorder

\section{Introduction}

Allergic rhinitis (AR) is a common chronic condition, and its symptoms, including nasal obstruction, repetitive sneezing, and watery nasal discharge, generally develop before the age of 20 years [1]. Approximately $40 \%$ of patients become symptomatic at 6 years of age, indicating that AR is a common health problem among adolescents [1]. Although symptoms are non-life-threatening, AR has been increasingly recognized to have a serious impact on patients' quality of life (QOL) [2]. The symptoms associated with AR in adolescents are different from those in children and adults, and have a more serious impact on their physical and mental conditions [3]. Sleep disorders, an AR-associated symptom [2], are more relevant in adolescents [3]. In addition, AR has a negative impact on social activities, including academic achievement [3]. Altogether, appropriate management of AR symptoms is particularly important in adolescents.

According to the guidelines for the treatment of AR, surgical treatments have been evaluated as an option to control severe nasal symptoms refractory to medical management $[2,4]$. Among the surgical treatments for AR, the efficiency of inferior turbinate (IT) surgeries, including submucosal resection of IT bones, has been well documented $[2,4]$. However, the impact of surgical treatments for AR on sleep and mental disorders in adolescents is not fully understood. In the current study, we retrospectively analyzed the efficacy of surgical treatment for AR in managing sleep and mental disorders in adolescents. 


\section{Methods}

\subsection{Subjects}

A retrospective chart review of patients aged 10-18 years who underwent endoscopic endonasal surgery for AR symptoms refractory to medical management, including oral antihistamines and topical steroids, from January 2015 to December 2016 at Kyoto ENT Surgicenter was performed. All patients were diagnosed perennial AR by the immunoglobulin E radioimmunosorbent test. The exclusion criteria were adenoid and/or tonsil hypertrophy and chronic sinusitis with nasal polyps and neoplasms. This study was approved by the institutional ethics committee (2017-5).

\subsection{Surgical Procedure}

All surgical procedures were performed endoscopically. All patients underwent bilateral submucosal IT bone resection. Briefly, a vertical incision was made on the anterior edge of the ITs, followed by dissection along the periosteum. After partial resection of the IT bones, the submucosal tissue was mildly cauterized through the periosteum. In general, posterior nasal neurectomy was performed simultaneously. Peripheral branches of the posterior nasal nerves were resected after being cauterized. Septoplasty was also done in cases with severe deviation of the nasal septum. During the septoplasty, the septal cartilage was preserved, and the vertical plate of the ethmoid and vomer was minimally resected.

\subsection{Outcomes Measured}

We identified 81 cases in which preoperative and postoperative (6 months after surgery) 22-item Sinonasal Outcome Test (SNOT-22) scores (Japanese version) [5] had been recorded. Twelve cases were excluded because of a lack of postoperative scores. SNOT-22 is a patient-reported outcome measure developed for use in chronic rhinosinusitis $[6,7]$. The SNOT-22 contains 22 individual questions with scores of 0 (no problem), 1 (very mild problem), 2 (mild or slight problem), 3 (moderate problem), 4 (severe problem), or 5 (problem as bad as it can be) for each question. We defined questions 1-4 (nasal to blow nose, nasal blockage, sneezing, and runny nose) as AR-associated questions. Questions 13-16 (difficulty falling sleep, waking up at night, lack of a good night's sleep, and waking up tired) were defined as sleep-associated questions. We also focused on questions 17-22 (fatigue, reduced productivity, reduced concentration, frustration, sadness, and embarrassment) as mental health questions.

We examined demographic data (age, sex, and surgical procedure) and preoperative and postoperative SNOT-22 and AR-, sleep-, and mental-associated questions. We analyzed correlations between the sums of preoperative scores for AR-associated questions (AR score), sleep-associated questions (sleep score) or mental health-associated questions (mental score), and between sleep and mental scores. To assess the surgical efficiency of AR-associated symptoms, we compared postoperative scores with preoperative scores in the SNOT-22 and AR scores. In addition, we validated the changes in SNOT-22 scores following surgical treatment based on the minimal clinically important difference (MCID), which is the smallest change in a treatment outcome that an individual patient would identify as important [8,9], for SNOT-22 in medically managed patients with chronic rhinosinusitis [10]. We then compared demographic characteristics, surgical procedures, and preoperative scores between subjects showing MCID for SNOT-22 and those showing smaller changes in SNOT-22 than MCID.

Next, we extracted subjects with a preoperative score of 3 (moderate problem) or more in both sleep-associated questions and mental health-associated questions. In these subjects, postoperative AR, sleep, and mental scores were compared with the preoperative scores. We then calculated the changes in AR, sleep, and mental scores (preoperative score-postoperative score) and analyzed the correlations among changes in AR, sleep, and mental scores. Furthermore, we divided the subjects into two groups according to surgical procedures performed, with or without septoplasty, and compared demographic characteristics, preoperative scores, and changes in scores after treatment. 


\subsection{Statistical Analyses}

The correlation was tested using the Spearman rank correlation. Comparisons between the preoperative and postoperative scores were performed using the Wilcoxon rank test. Other comparisons between two groups were tested using Fisher's exact test for nominal variables or Mann-Whitney U-test for continuous variables. Statistical analyses were performed using GraphPad Prism 7 (GraphPad Software, San Diego, CA, USA). Statistical significance was set at $p<0.05$. The data are presented as the mean \pm one standard deviation.

\section{Results}

\subsection{Total Subjects}

The subjects included 17 women and 64 men. The mean age was $14.1 \pm 2.0$ years. In $79 / 81$ patients, posterior nasal neurectomy was performed. In $42 / 81$ patients, septoplasty was also performed due to severe deviation of the nasal septum. No postoperative complications were found in these subjects. Preoperative SNOT-22, AR, sleep, and mental scores were $34.9 \pm 18.4$ (22 items), $11.8 \pm 5.0$ ( 4 items), $5.4 \pm 4.9$ (4 items), and $8.7 \pm 7.3$ (6 items), respectively. The correlation between preoperative AR and sleep scores, preoperative $\mathrm{AR}$ and mental scores, and preoperative sleep and mental scores were statistically significant (Figure $1 ; p=0.004, \mathrm{r}=0.32$ for AR and sleep scores; $p<0.0001, \mathrm{r}=0.44$ for AR and mental scores; $p<0.0001, \mathrm{r}=0.66$ for sleep and mental scores).

A

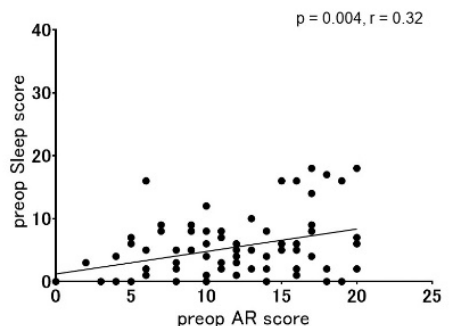

B

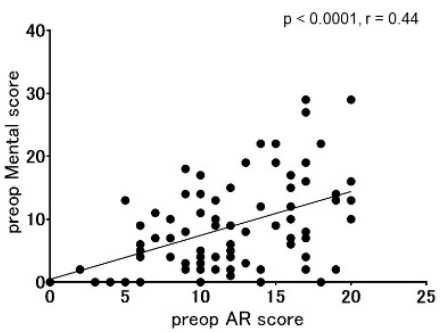

C

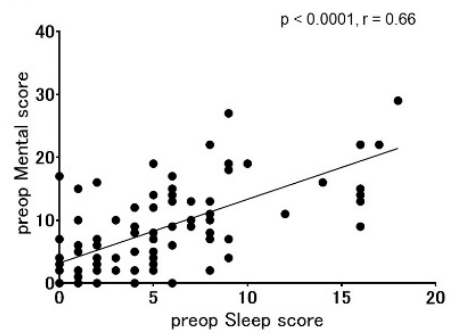

Figure 1. Correlations between preoperative AR, sleep, and mental scores in all the subjects. (A) correlation between AR and sleep scores, (B) correlation between AR and mental scores, (C) correlation between sleep and mental scores. Significant correlations among AR, sleep, and mental scores were observed with the Spearman rank correlation $(p=0.004, \mathrm{r}=0.32$ for AR and sleep scores; $p<0.0001$, $\mathrm{r}=0.44$ for AR and mental scores; $p<0.0001, \mathrm{r}=0.66$ for sleep and mental scores).

Postoperative SNOT-22 and AR scores were $14.5 \pm 15.9$ and $5.5 \pm 5.0$, respectively, and were significantly improved in comparison with preoperative scores ( $p<0.0001$ for each). The medians and $95 \%$ confidence intervals (CIs) for differences between preoperative and postoperative scores were 20.4 (median) and 16.5-24.2 (95\% CI) for SNOT-22 (22 items) and 6.3 (median) and 5.0-7.6 (95\% CI) for AR scores (4 items).

A recent study has indicated that the MCID for SNOT-22 in medically managed patients with chronic rhinosinusitis is a 12-point [10]. The proportion of subjects showing MCID (improvement of 12 or larger points) for SNOT-22 in our subjects was 72.8\% (59/81 patients). Table 1 shows the demographic characteristics, surgical procedures, and preoperative scores in subjects with or without MCID. No significant difference in sex, age, or surgical procedure (with or without septoplasty) was found between subjects with MCID and those without MCID (Table 1). All preoperative scores in subjects with MCID were significantly higher than those in subjects without MCID (Table 1). 
Table 1. Demographic characteristics, surgical procedures, and preoperative scores in subjects with or without MCID. Asterisks $\left(^{*}\right)$ indicate statistical significance by the Mann-Whitney U-test.

\begin{tabular}{cccc}
\hline$\cdot$ & MCID $+(n=59)$ & MCID $-(n=\mathbf{2 2})$ & Statistics \\
\hline Sex (woman:man) & $10: 49$ & $7: 15$ & $p=0.2180$ \\
\hline Age (years) & $13.9 \pm 1.9$ & $14.9 \pm 2.1$ & $p=0.0587$ \\
\hline $\begin{array}{c}\text { Surgical procedure } \\
\text { (with: without septoplasty) }\end{array}$ & $30: 29$ & $12: 10$ & $p=0.8071$ \\
\hline Preoperative SNOT-22 & $38.8 \pm 15.7$ & $24.4 \pm 21.7$ & ${ }^{*} p<0.0001$ \\
\hline Preoperative AR score & $12.7 \pm 4.4$ & $9.5 \pm 5.8$ & ${ }^{*} p=0.0361$ \\
\hline Preoperative Sleep score & $6.3 \pm 4.7$ & $3.1 \pm 4.7$ & ${ }^{*} p=0.0005$ \\
\hline Preoperative Mental score & $10.2 \pm 6.7$ & $4.5 \pm 7.4$ & ${ }^{*} p<0.0001$ \\
\hline
\end{tabular}

\subsection{Subjects with Sleep and Mental Disorders}

Sleep and mental disorders were identified in 34 subjects (6 women). The mean age was $13.7 \pm 2.0$ years. Septoplasty was performed in 16 patients $(47.1 \%)$. The preoperative and postoperative SNOT-22 were $48.7 \pm 17.7$ and $17.8 \pm 20.5$, respectively. The difference was statistically significant at $p<0.0001$. The mean and $95 \%$ CI of differences between preoperative and postoperative SNOT-22 was 30.9 and 25.0-36.8. The proportion of subjects with MCID (improvement of 12 or larger points) was $94.1 \%$ (32/34 patients).

The preoperative and postoperative AR, sleep, and mental scores of these subjects are shown in Figure 2. In all domains, postoperative scores were significantly reduced in comparison with the preoperative scores $(p<0.0001$ for each). The average changes between preoperative and postoperative scores for 1 item were 2.0 (95\% CI: 1.5-2.5) for AR scores, 1.6 (95\% CI: 1.3-1.9) for sleep scores, and 1.6 (95\% CI: 1.3-2.0) for mental scores. Correlations between changes in AR scores and mental scores and between those in sleep scores and mental scores were statistically significant (Figure 3B,C; $p=0.0028, \mathrm{r}=0.50$ for $\mathrm{AR}$ and mental scores; $p<0.0001, \mathrm{r}=0.63$ for sleep and mental scores). No significant correlation was observed between changes in AR scores and those in sleep scores (Figure 3A; $p=0.14, \mathrm{r}=0.25)$.

A

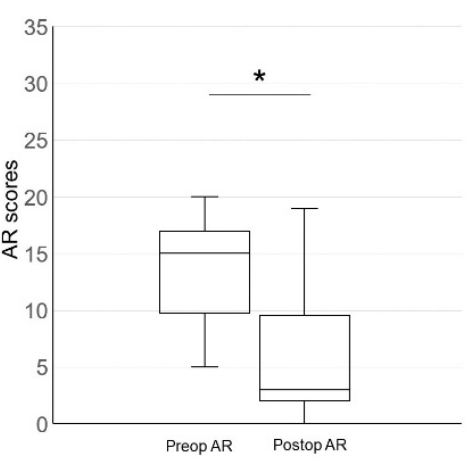

B

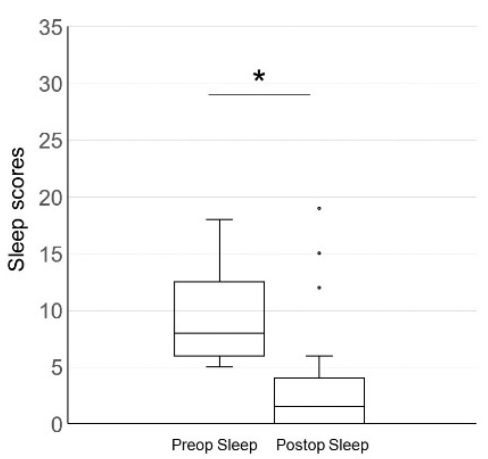

C

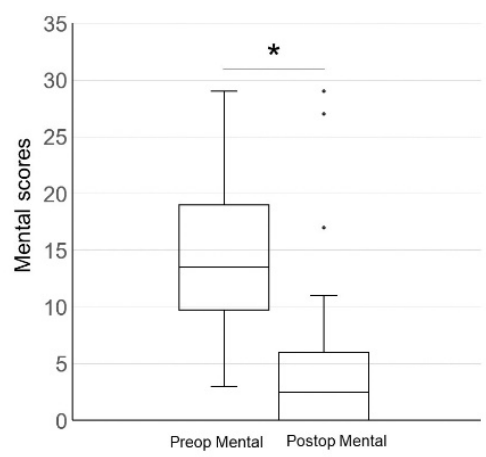

Figure 2. Box plots of preoperative and postoperative AR (A), sleep (B), and mental scores (C) in patients with both sleep and mental disorders. Differences between the preoperative and postoperative scores for each parameter were statistically significant at $p<0.0001$ using the Wilcoxon rank test $\left(^{*}\right)$. 
A

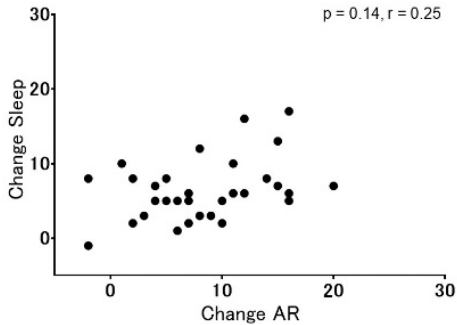

B

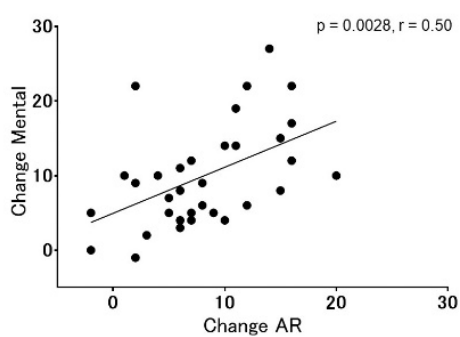

C

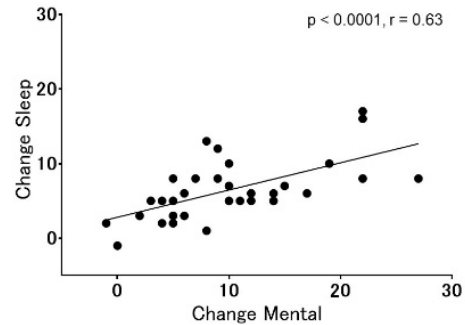

Figure 3. Correlations between changes in AR scores and those in sleep scores (A), between changes in AR scores and those in mental scores (B), and between changes in sleep scores and those in mental scores $(\mathbf{C})$. A significant correlation was identified between AR and mental scores $(p=0.0028, \mathrm{r}=0.50)$ or between sleep and mental scores $(p<0.0001, \mathrm{r}=0.63)$ with Spearman rank correlation.

To test the effects of septoplasty on outcomes, subjects were divided into two groups: subjects that underwent septoplasty (with septoplasty) and those that did not (without septoplasty). Demographic characteristics and preoperative scores in two groups are shown in Table 2. There were no significant differences in sex, age, or preoperative scores between the two groups (Table 2), indicating no significant differences in the baseline between the two groups. For the changes in SNOT-22, AR, sleep, or mental scores after treatment, no significant differences were identified between subjects with septoplasty and those without septoplasty, although there was a trend of larger changes in the AR scores in subjects without septoplasty (Table 3).

Table 2. Demographic characteristics and preoperative scores in subjects showing sleep and mental disorders with or without septoplasty.

\begin{tabular}{cccc}
\hline & $\begin{array}{c}\text { With Septoplasty } \\
(\boldsymbol{n}=\mathbf{1 6})\end{array}$ & $\begin{array}{c}\text { Without Septoplasty } \\
(\boldsymbol{n}=\mathbf{1 8})\end{array}$ & Statistics \\
\hline Sex (woman:man) & $3: 13$ & $1: 17$ & $p=0.3226$ \\
\hline Age (years) & $14.1 \pm 2.1$ & $13.3 \pm 1.9$ & $p=0.3445$ \\
\hline Preoperative SNOT-22 & $45.2 \pm 17.1$ & $51.8 \pm 18.2$ & $p=0.2202$ \\
\hline Preoperative AR score & $13.8 \pm 4.6$ & $13.5 \pm 4.3$ & $p=0.7388$ \\
\hline Preoperative sleep score & $9.9 \pm 4.4$ & $8.6 \pm 4.3$ & $p=0.2802$ \\
\hline Preoperative mental score & $13.2 \pm 7.0$ & $15.4 \pm 6.3$ & $p=0.3875$ \\
\hline
\end{tabular}

Table 3. Changes in SNOT-22, AR, sleep, and mental scores in subjects showing sleep and mental disorders with or without septoplasty.

\begin{tabular}{ccccc}
\hline & $\begin{array}{c}\text { Changes in } \\
\text { SNOT-22 }\end{array}$ & $\begin{array}{c}\text { Changes in } \\
\text { AR Scores }\end{array}$ & $\begin{array}{c}\text { Changes in } \\
\text { Sleep Scores }\end{array}$ & $\begin{array}{c}\text { Changes in } \\
\text { Mental Scores }\end{array}$ \\
\hline with septoplasty & $28.6 \pm 18.7$ & $6.3 \pm 5.4$ & $6.2 \pm 4.2$ & $10.2 \pm 6.2$ \\
\hline without septoplasty & $33.4 \pm 14.8$ & $10.0 \pm 5.0$ & $6.6 \pm 3.7$ & $9.6 \pm 6.2$ \\
\hline Statistics & $p=0.4168$ & $p=0.0530$ & $p=0.8703$ & $p=0.7136$ \\
\hline
\end{tabular}

\section{Discussion}

Previous studies have indicated that adolescents differ from adults and children in terms of the degree of their AR symptoms and their impact on QOL [3]. Among AR symptoms, nasal obstruction is more bothersome in adolescents than in adults or children and has a greater effect on QOL in adolescents than in children in a French study of seasonal AR symptoms [11]. A Japanese survey of a large working population demonstrated a high degree of nasal obstruction in young subjects and a relationship between nasal obstruction 
and daytime sleepiness [12]. A real-life prospective study of Spanish adolescents revealed a strong correlation between worse nasal obstruction and worse QOL [13]. Importantly, the burden of AR on education in adolescents has also been reported [14-16]. These findings indicate that the impact of controlling AR symptoms on QOL can be greater in adolescents than in adults or children.

Surgical treatment is an option to control severe nasal symptoms and to improve QOL in AR patients refractory to medical management [2,4]. IT is a primary target of allergic effects. Therefore, IT reduction is a main stream of surgical options for AR [2,4], although its efficacy for AR symptoms is still controversial [17]. Among numerous techniques for IT reduction $[18,19]$, submucosal bony resection is a traditional procedure and its long-term efficacy for maintenance of nasal airflow is known [20]. Another merit of submucosal bony resection is the preservation of the surface mucosa, which contributes to the maintenance of ciliary function [18]. To reduce the risk for atrophic rhinitis, which is one complication after submucosal bony resection [18], we performed partial resection of IT bones. Posterior nasal neurectomy is a modified surgical technique of vidian neurectomy, which decreases hypersecretion and hypersensitivity [21]. Based on this, a combination of submucosal resection of IT bones and posterior nasal neurectomy has been our standard surgery used for AR patients refractory to medical management.

In contrast to IT, the nasal septum is not a major contributor to allergic disease. However, to achieve a reduction of subjective nasal obstruction in AR patients, septoplasty is required for AR patients with severe septal deviation [2,18]. Based on this thesis, we performed septoplasty in addition to IT surgery in selected cases. In this study, half of the subjects underwent septoplasty with IT surgery. In the present results, septoplasty had no significant effect on achievement of the MCID for SNOT-22 after surgery in the total subjects, or on changes in SNOT-22, AR, sleep, or mental scores after surgery in subjects showing sleep and mental disorders. We consider that these results may not be associated with poor effects of septoplasty on AR symptoms. In the present study, we performed septoplasty only in selected patients with severe septal deviation, which represents a bias within the validation of outcomes.

The current study examined the effects of surgical treatment for AR on sleep and mental disorders in adolescents, which could contribute to the determination of surgical indications for AR in adolescents. The present results in all subjects demonstrated significant correlations between preoperative AR, sleep, and mental scores in adolescents with AR refractory to medical management, suggesting that severe AR symptoms can be a cause for sleep and mental disorders in adolescents, which is identical to previous studies [3]. The surgical modality used in this study significantly reduced the SNOT-22 and AR scores, and SNOT-22 improvement of 12 or more points, which is the MCID for SNOT-22 in chronic rhinosinusitis patients [10], was found in $72.8 \%$ of subjects. These suggests the efficacy of our surgical modality in controlling AR symptoms. Subjects with MCID exhibited higher preoperative SNOT-22, AR, sleep, and mental scores than those without MCID, indicating that the impact of surgical treatment was larger in patients with severe symptoms than those with slight or moderate symptoms.

To validate the effects of surgical treatment on sleep and mental disorders in adolescents, we extracted 34 patients who had at least moderate problems in both sleep and mental questions, which was $42.0 \%$ of the total subjects. This indicates a high incidence of sleep and mental problems in adolescents with AR refractory to medical management. Surgical treatment significantly improved AR, sleep, and mental scores in these subjects. In addition, in over $90 \%$ subjects, an improvement in SNOT-22 scores better than the MCID for SNOT-22 [10] was achieved, suggesting high impacts of surgical treatment on subjective symptoms in adolescent patients with sleep and mental disorders. The improvement in the AR score was significantly correlated with that in the mental score, indicating a close relationship between AR symptoms and mental disorders in adolescents with AR-refractory medical management. The improvement in sleep scores also showed a significant and high correlation with mental scores. Altogether, the present findings indicate that severe 
AR cases with sleep and/or mental disorders represent a good indication for surgical intervention, especially in adolescents. However, the outcomes measured in this study lacked objective assessments of surgical effects. Therefore, distinct effects of our surgical modality on the improvement of nasal obstruction were unclear. In future studies, the effects of alterations in the nasal airflow that are determined by objective assessments of sleep and mental disorders should be examined.

\section{Conclusions}

In the present study, we retrospectively analyzed the effects of surgical treatment on sleep and mental disorders in adolescents with AR symptoms refractory to medical management, which indicates the efficacy of surgical treatment not only for AR symptoms but also for sleep and mental disorders. Considering the particular impact of AR symptoms on QOL in adolescents, surgical intervention is a recommended treatment option in patients with sleep or mental disorders.

Supplementary Materials: The following supporting information can be downloaded at: https: //www.mdpi.com/article/10.3390/surgeries3010005/s1.

Author Contributions: T.N. conceptualized, designed, and performed the study and wrote the manuscript. E.O.-N. and S.H. acquired the data. All authors have read and agreed to the published version of the manuscript.

Funding: This research received no external funding.

Institutional Review Board Statement: The study was conducted according to the guidelines of the Declaration of Helsinki, and approved by the Institutional Review Board of Kyoto ENT Surgicenter (protocol code 2017-5, date of approval 29 November 2017).

Informed Consent Statement: Informed consent was obtained from all subjects included in the study.

Data Availability Statement: Data is contained within the supplementary material.

Conflicts of Interest: The authors have no conflict of interest directly relevant to the content of this article.

\section{References}

1. Meltzer, E.O.; Blaiss, M.S.; Derebery, M.J.; Mahr, T.A.; Gordon, B.R.; Sheth, K.K.; Simmons, A.L.; Wingertzahn, M.A.; Boyle, J.M. Burden of allergic rhinitis: Results from the Pediatric Allergies in America survey. J. Allergy Clin. Immunol. 2009, 124, S43-S70. [CrossRef] [PubMed]

2. Wise, S.K.; Lin, S.Y.; Toskala, E.; Orlandi, R.R.; Akdis, C.A.; Alt, J.A.; Azar, A.; Baroody, F.M.; Bachert, C.; Canonica, G.W.; et al. International consensus statement on allergy and rhinology: Allergic rhinitis. Int. Forum. Allergy Rhinol. 2018, 8, 108-352. [CrossRef] [PubMed]

3. Blaiss, M.S.; Hammerby, E.; Robinson, S.; Kennedy-Martin, T.; Buchs, S. The burden of allergic rhinitis and allergic rhinoconjunctivitis on adolescents: A literature review. Ann. Allergy Asthma. Immunol. 2018, 121, 43-52. [CrossRef] [PubMed]

4. Seidman, M.D.; Gurgel, R.K.; Lin, S.Y.; Schwartz, S.R.; Baroody, F.M.; Bonner, J.R.; Dawson, D.E.; Dykewicz, M.S.; Hackell, J.M.; Han, J.K.; et al. AAO-HNSF. Clinical practice guideline: Allergic rhinitis. Otolaryngol. Head Neck Surg. 2015, 152 (Suppl. 1), S1-S43. [CrossRef]

5. Ogino-Nishimura, E.; Hiroshiba, S.; Iwanaga, M. Reliability, validity and responsiveness of the Sino-Nasal Outcome Test (SNOT-22) in Japanese patients. Nippon. Jibiinkoka Gakkai Kaiho 2017, 120, 1155-1164. [CrossRef]

6. Piccirillo, J.F.; Merritt, M.G., Jr.; Richards, M.L. Psychometric and clinimetric validity of the 20-Item Sino-Nasal Outcome Test (SNOT-20). Otolaryngol. Head Neck Surg. 2002, 126, 41-47. [CrossRef]

7. Hopkins, C.; Gillett, S.; Slack, R.; Lund, V.J.; Browne, J.P. Psychometric validity of the 22-item Sinonasal Outcome Test. Clin. Otolaryngol. 2009, 34, 447-454. [CrossRef]

8. Jaeschke, R.; Singer, J.; Guyatt, G.H. Measurement of health status. Ascertaining the minimal clinically important difference. Control. Clin. Trials 1989, 10, 407-415. [CrossRef]

9. Wright, A.; Hannon, J.; Hegedus, E.J.; Kavchak, A.E. Clinimetrics corner: A closer look at the minimal clinically important difference (MCID). J. Man Manip. Ther. 2012, 20, 160-166. [CrossRef]

10. Phillips, K.M.; Houssein, F.A.; Boeckermann, L.M.; Singerman, K.W.; Liu, D.T.; Sedaghat, A.R. Multi-institutional minimal clinically important difference of the 22-item Sinonasal Outcome Test in medically managed chronic rhinosinusitis. Rhinology 2021, 59, 552-559. [CrossRef] 
11. Devillier, P.; Bousquet, P.J.; Grassin-Delyle, S.; Salvator, H.; Demoly, P.; Bousquet, J.; de Beaumont, O. Comparison of outcome measures in allergic rhinitis in children, adolescents and adults. Pediatr. Allergy Immunol. 2016, 27, 375-381. [CrossRef] [PubMed]

12. Udaka, T.; Suzuki, H.; Kitamura, T.; Shiomori, T.; Hiraki, N.; Fujimura, T.; Ueda, N. Relationships among nasal obstruction, daytime sleepiness, and quality of life. Laryngoscope 2006, 116, 2129-2132. [CrossRef]

13. Valls-Mateus, M.; Marino-Sanchez, F.; Ruiz-Echevarría, K.; Cardenas-Escalante, P.; Jiménez-Feijoo, R.; Blasco-Lozano, J.; GinerMuñoz, M.T.; Haag, O.; Alobid, I.; Plaza Martin, A.M.; et al. Nasal obstructive disorders impair health-related quality of life in adolescents with persistent allergic rhinitis: A real-life study. Pediatr. Allergy Immunol. 2017, 28, 438-445. [CrossRef]

14. Devillier, P.; Bousquet, J.; Salvator, H.; Naline, E.; Grassin-Delyle, S.; de Beaumont, O. In allergic rhinitis, work, classroom and activity impairments are weakly related to other outcome measures. Clin. Exp. Allergy 2016, 46, 1456-1464. [CrossRef]

15. Meltzer, E.O.; Farrar, J.R.; Sennett, C. Findings from an online survey assessing the burden and management of seasonal allergic rhinoconjunctivitis in US patients. J. Allergy Clin. Immunol. Pract. 2017, 5, 779-789. [CrossRef] [PubMed]

16. Sundberg, R.; Torén, K.; Höglund, D.; Aberg, N.; Brisman, J. Nasal symptoms are associated with school performance in adolescents. J. Adolescent Health 2007, 40, 581-583. [CrossRef]

17. Jose, J.; Coatesworth, A.P. Inferior turbinate surgery for nasal obstruction in allergic rhinitis after failed medical treatment. Cochrane Database Syst. Rev. 2010, 8, CD005235. [CrossRef]

18. Chhabra, N.; Houser, S.M. Surgery for allergic rhinitis. Int. Forum. Allergy Rhinol. 2014, 4 (Suppl. 2), S79-S83. [CrossRef] [PubMed]

19. Brunworth, J.; Holmes, J.; Sindwani, R. Inferior turbinate hypertrophy: Review and graduated approach to surgical management. Am. J. Rhinol. Allergy 2013, 27, 411-415. [CrossRef]

20. Mori, S.; Fujieda, S.; Yamada, T.; Kimura, Y.; Takahashi, N.; Saito, H. Long-term effect of submucous turbinectomy in patients with perennial allergic rhinitis. Laryngoscope 2002, 112, 865-869. [CrossRef]

21. Kobayashi, T.; Hyodo, M.; Nakamura, K.; Komobuchi, H.; Honda, N. Resection of peripheral branches of the posterior nasal nerve compared to conventional posterior neurectomy in severe allergic rhinitis. Auris Nasus Larynx 2012, 39, 593-596. [CrossRef] [PubMed] 